17.1

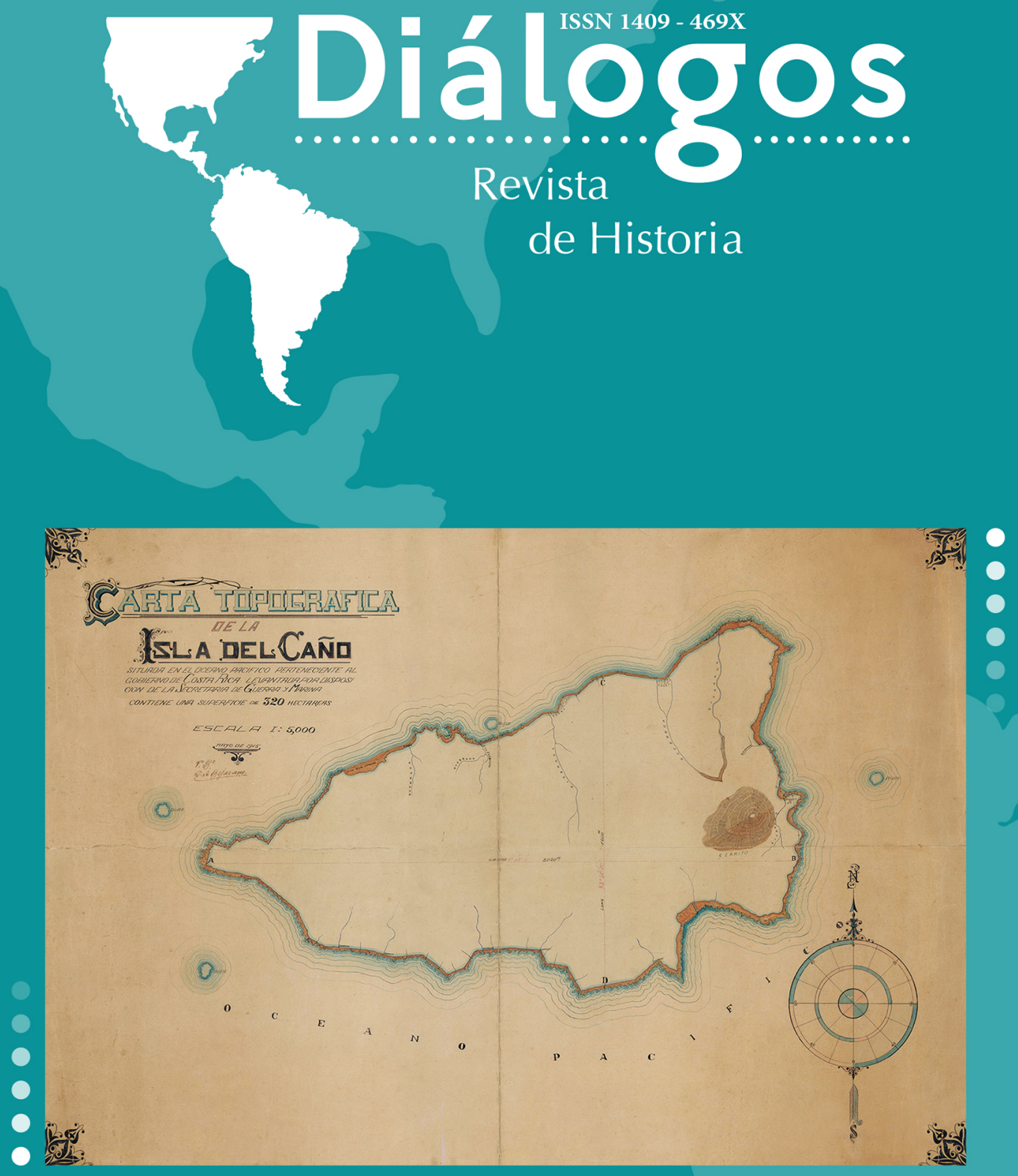

Escuela de Historia. Universidad de Costa Rica

Enero-junio 2016

url: http://revistas.ucr.ac.cr/index.php/dialogos/index 


\title{
LA CARTOTECA HISTÓRICA DE COSTA RICA, UN MEDIO PARA LA PROTECCIÓN Y DIVULGACIÓN DEL PATRIMONIO CARTOGRÁFICO
}

\author{
Adolfo Quesada Román \\ Gustavo Barrantes Castillo
}

\begin{abstract}
Resumen
Las colecciones de mapas y planos históricos que conserva el Archivo Nacional de Costa Rica son de invaluable valor cultural y debido a su fragilidad, deben ser preservados en formato digital para su manipulación. La iniciativa de crear una Cartoteca Histórica de Costa Rica, promueve no solo la protección de estos documentos, sino también su divulgación electrónica. Entre sus principales productos potenciales están los insumos para investigación científica, la consulta en general y la promoción de la geografía histórica.
\end{abstract}

Palabras claves: cartografía, Costa Rica, historia, geografía, geografía histórica.

\section{HISTORICAL MAP LIBRARY OF COSTA RICA, A MEDIUM FOR CARTOGRAPHIC HERITAGE PROTECTION AND DIVULGATION}

\begin{abstract}
Collections of historical maps which preserve the National Archives of Costa Rica have an invaluable cultural value and due to its fragility, should be preserved in digital format for it manipulation. The initiative for create a Historic Map Library of Costa Rica, not only promotes the protection of these documents but also its electronic divulgation. Its main products are potential inputs for scientific research, consultation in general and the promotion of historical geography.
\end{abstract}

Keywords: cartography, Costa Rica, history, geography, historical geography.

Fecha de recepción: 23 de marzo del 2015 • Fecha de aceptación: 15 de julio 2015

\footnotetext{
Adolfo Quesada Román • Costarricense. Egresado de la Maestría en Geografía Ambiental - de la Universidad Nacional Autónoma de México (UNAM). Docente e investigador de la

- Escuela de Ciencias Geográficas de la Universidad Nacional de Costa Rica (UNA). Correo

- electrónico: adolfo.quesada@gmail.com

-

Gustavo Barrantes Castillo - Costarricense. Geógrafo, Máster en Computación por el Instituto Tecnológico de Costa Rica (ITCR). Docente e investigador de la Escuela de

- Ciencias Geográficas de la Universidad Nacional de Costa Rica (UNA). Correo electrónico:

gustavo.barrantes.castillo@una.cr
} 


\section{INTRODUCCIÓN}

El conocimiento del espacio ha sido fundamental en el desarrollo de la civilización, sus implicaciones han sido estratégicas desde los primeros días de la civilización humana, tanto para ubicar puntos por medio de las estrellas como para localizar sitios de caza durante las grandes migraciones. La representación del espacio es realizada por medio de mapas, los cuales conforme ha pasado el tiempo, han perfeccionado sus técnicas y precisión. Los mapas tienen múltiples funciones y detrás de su confección hay otras connotaciones, como la geopolítica o socioeconómica.

Durante el periodo de conquista y colonización de América, por ejemplo, el conocimiento de las costas, la ubicación de asentamientos indígenas, de recursos mineros, de cursos fluviales, fueron elementos centrales que impulsaron la cartografía de la época, por parte de la corona española. Es por ello que se tiene un acervo importante de cartografía político-administrativa desde el siglo XVI, en el cual han quedado representados los países del área en diferentes etapas históricas.

Los mapas que a lo largo del tiempo se han creado en Costa Rica, ya sea para representar las ciudades, la distribución de las carreteras o la orografía, los límites territoriales, entre otros, y que alcanzaron un carácter público, se hicieron parte de los archivos oficiales del Estado. Todos estos archivos han quedado bajo el resguardo del Archivo Nacional de Costa Rica, que es el ente gubernamental oficial para esta tarea.

La historia del Archivo Nacional de Costa Rica puede ser explicada en tres etapas. La primera etapa corresponde con su fundación y de replanteamiento, la cual se ubica entre los años 1881-1888, caracterizándose por presentar documentos desorganizados, incompletos y deteriorados, así como por el reducido número de personal e instalaciones inadecuadas. La segunda etapa se extiende de 1889 a 1957 cuando el Archivo Nacional es instalado en el edificio de la Universidad de Santo Tomás (Murillo-Delgado, 1981).

Durante este periodo se inicia la organización de sus materiales y la elaboración de ficheros para su consulta. En 1902 se dicta el Primer Reglamento de los Archivos Nacionales, en el cual se dan lineamientos técnicos para la clasificación y la ordenación de los documentos. En 1934 se crea un timbre de archivo con la finalidad de construir un edificio adecuado para los Archivos Nacionales, pero es hasta 50 años después, en 1993, cuando se inaugura la primera etapa de su edificio. En 1936 se edita la Revista del Archivo Nacional, cuyo objetivo era difundir los fondos documentales en custodia, así como las investigaciones históricas (Murillo-Delgado, 1981).

El periodo de replanteamiento (1969-1990) se caracteriza por un mayor respaldo de las autoridades gubernamentales y por una gran cooperación internacional, sobre todo de la Organización de Estados Americanos (OEA) y de la UNESCO, que permitieron la capacitación del personal del Archivo Nacional en el exterior, principalmente en Argentina y España, con lo cual su personal adquiere nuevos conocimientos que permiten obtener una nueva visión del quehacer archivístico. 
Asimismo, por medio de la Ley 5574 del 17 de setiembre de 1974 se crea una junta administrativa con nuevas funciones, principalmente la de dotar al Archivo Nacional de un edificio propio. Además, se crea una fuente de financiamiento permanente mediante un impuesto a los cheques y los recursos obtenidos por el timbre de archivos (Archivo Nacional de Costa Rica, 2014).

A las etapas anteriores, se les debe agregar una cuarta etapa que se podría llamar de consolidación, que se inicia en 1990 con la creación de la Ley 7202 del Sistema Nacional de Archivos. Mediante esa ley se dota al Archivo Nacional de un marco jurídico que lo convierte en el Archivo Histórico más grande del país y en el ente rector del Sistema Nacional de Archivos. Asimismo, se logra el sueño acariciado desde 1934 de dotarlo de un edificio moderno con las condiciones adecuadas para conservar el patrimonio documental de Costa Rica. Durante esta etapa, el Archivo Nacional ha logrado destacarse tanto en el ámbito nacional como internacional, principalmente en el Consejo Internacional de Archivos y en la Asociación Latinoamericana de Archivos (Quesada-Camacho, 2001).

Con la creación del Archivo Nacional se desarrolla una institución que, entre otros servicios, debe velar por la integridad física de estos documentos ahora de carácter histórico. Los materiales resguardados por esta institución cobran un valor inestimable debido a su importancia como documentos de primera referencia para estudios históricos, etnogeográficos y geopolíticos, entre otros; no obstante, su consulta implica un riesgo de daño, ya que sus materiales son muy susceptibles a sufrir deterioro físico, lo que representa un problema que requiere de una solución innovadora.

Con el advenimiento de las Tecnologías de Información y Comunicación (TIC) se ha hecho posible trasladar la información en formato papel a formato digital y de esta forma facilitar su acceso desde cualquier ubicación, ya sea dentro del país, en la región Centroamericana o en el mundo, para bien del valor histórico y la cultura en general.

Desde hace varias décadas son comunes las mapotecas o cartotecas dentro de las universidades, museos y bibliotecas, especialmente en los países desarrollados, las cuales han empezado a adoptar tecnologías que les permiten migrar sus colecciones al formato digital. En América Latina resaltan las mapotecas de México, Argentina, Chile, Colombia y otros países latinoamericanos, los cuales poseen una larga tradición cartográfica; algunas de ellas ya han comenzado su migración hacia las mapotecas virtuales, ofreciendo acceso a partes de su colección.

Desde el 2007, la Escuela de Ciencias Geográficas de la Universidad Nacional de Costa Rica (UNA) desarrolló un sitio web denominado la Mapoteca Virtual, el cual consiste en una plataforma de software libre que ha sido adaptado para almacenar mapas digitales en distintos formatos, así como ofrecer funcionalidades de búsqueda y descarga, contenidos relacionados con la cartografía y un banner a modo de portal de cartografía para Costa Rica (Barrantes, Tercero, Picado y Morera, 2010). Actualmente cuenta con una amplia colección: mapas digitales producidos por diversas instituciones 
públicas y especialistas de la Universidad Nacional (UNA), manuales sobre técnicas cartográficas y de uso de Sistemas de Información Geográfica (SIG) y una muestra de productos académicos relacionados con la cartografía y el software libre SIG. Los objetivos de esta iniciativa son (Barrantes, 2011):

1 Proveer material digital que promueva la incorporación de las TIC en el proceso de enseñanza-aprendizaje.

2 Acercar a los investigadores por medio de la difusión de sus productos cartográficos.

3 Facilitar el uso y acceso libre de materiales relacionados con la cartografía y la geografía a estudiantes de primaria y secundaria, universitarios y para el público en general.

Como parte de las actividades académicas del proyecto de la Mapoteca Virtual, se buscaron alianzas estratégicas para la divulgación de información cartográfica del país. Se logró un contacto recíproco con el Archivo Nacional, la institución que tutela la mayor cantidad de mapas históricos en el país.

Como resultado se inició la redacción de un convenio específico entre la UNA y de la DGAN (Dirección General del Archivo Nacional) que fue firmado en 2013. Dicho acuerdo contempla la facilitación de un escáner de alta resolución por parte de la Escuela de Ciencias Geográficas así como la participación de estudiantes del Diplomado de Cartografía y Diseño Digital en calidad de asistentes académicos, con el fin de escanear los mapas y planos antiguos; a cambio, el Área de Conservación del Archivo Nacional se comprometió a brindar un espacio de trabajo y la colección de mapas y planos para ponerlos a disposición del público en general, a través del portal de la Mapoteca Virtual. En total, se pretende escanear alrededor de 10000 mapas y planos del acervo histórico existente.

La importancia de proteger este acervo cultural, a la vez que hacerlo accesible a investigadores, estudiantes y amantes de la cartografía ha llevado al desarrollo de una Cartoteca Histórica de Costa Rica y un marco de colaboración entre las dos instituciones. Este vínculo es un paso importante en el desarrollo conjunto entre instituciones públicas del país para integrar iniciativas que sean de beneficio para los dos entes interesados, la comunidad científica y el público en general. En el marco de este proyecto, se hace énfasis en la colaboración de estudiantes del Diplomado en Cartografía y Diseño Digital y del Bachillerato de Ciencias Geográficas, de la Escuela de Ciencias Geográficas de la UNA, para realizar su práctica profesional a través del desarrollo de atlas históricos temáticos.

Vale resaltar que antes de la puesta en vigencia de este convenio, el archivo utilizaba una cámara fotográfica que colocada en una estructura metálica que le permitía suspenderse sobre una mesa, de esta forma fotografiaban los planos o mapas originales a solicitud de los interesados, quienes los utilizaban para zanjar disputas legales o para realizar estudios históricos. 


\section{METODOLOGÍA}

Para el desarrollo de este trabajo, fue necesario realizar una selección minuciosa de mapas o planos que a nuestro criterio tienen valor para la Geografía Histórica, particularmente los que tengan relación con centros urbanos, usos del suelo, formas del terreno y actividades económicas. De modo que se han excluido los planos constructivos u otros documentos que no tengan relación con los objetivos de la Mapoteca Virtual.

El procedimiento a seguir se resume en los siguientes pasos:

1 Revisión del material cartográfico almacenado en cada carpeta del Archivo Nacional (AN), las cuales constan de alrededor de 50 ejemplares cada una.

2 Se seleccionan los materiales que tengan valor histórico-geográfico.

3 Se levanta una ficha de metadato (información que define las características de un archivo digital) con los datos más relevantes de cada pieza.

4 Se escanea el material seleccionado, se asocia con su metadato y su ubicación en la colección del AN, por medio de un número de signatura.

5 Se almacena el archivo escaneado en alta calidad en la base de datos de la DGAN.

6 Se lleva una copia de la base de datos a la UNA, que se almacena en el Repositorio de la Universidad Nacional de Costa Rica (http://www.repositorio.una.ac.cr/).

7 Se abre un repositorio exclusivo para la búsqueda y almacenamiento de la cartografía histórica que próximamente se pondría a disposición del público, en cuanto se reúna un lote inicial de 1500 ejemplares.

\section{RESULTADOS}

Entre los resultados alcanzados, actualmente se tienen más de 1200 mapas y planos que representan un aporte significativo en la protección de dichos materiales, pues de ahora en adelante quien los requiera podrá accederlos en formato digital en el Archivo Nacional y próximamente en la Mapoteca Virtual, a través del repositorio denominado Cartoteca Histórica.

Otro producto resultante consiste en la elaboración de metadatos, estos siguen una estandarización y clasificación ordenada de la información cartográfica; un ejemplo de ellos es el ISO 19115: Geographic information - Metadata (International Standards Organization, 2014). Entre las variables que conforman el archivo de metadatos utilizado en este trabajo, están las siguientes: Nombre, título, descripción, colección, cobertura, autor o autores, fecha de creación, fecha de publicación, fecha de modificación, formato, idioma, fuente, descriptores, tipo de recurso, capas, contacto-recurso, coordenadas extremas, escala, exactitud, formato mapa, modelo de dato, precisión, proyección, licencia de uso, sistema de referencia, software y tema. Mediante estos datos, cada mapa tendrá una referencia espacial, 
temporal y de autoría. Todo esto redundará en más que una mejor indexación que facilite la búsqueda, en una base de datos completa, en una forma de difundir los mapas con una referencia adecuada para ser citados en otras investigaciones.

Se ha desarrollado un repositorio específico para almacenar esta colección dentro del Programa UNA WEB (Cartoteca Histórica), unidad que se encargará del soporte técnico y de su mantenimiento. Además, esta iniciativa brindará las funciones de búsqueda y descarga orientada por estándares de Open Access y software libre, las cuales son herramientas útiles para el acceso de la información cartográfica histórica de manera segura y pronta para diferentes grupos interesados, entre ellos bibliotecarios, investigadores (historiadores, educadores, geógrafos, ambientalistas, arquitectos u otros) y administradores académicos (Reinsfelder y Anderson, 2013).

Es una prioridad para el acervo histórico de la cartografía de Costa Rica, que se utilice todo su potencial mediante un archivado, catalogación y distribución eficientes de la información geoespacial, que puede ser usada por el sector

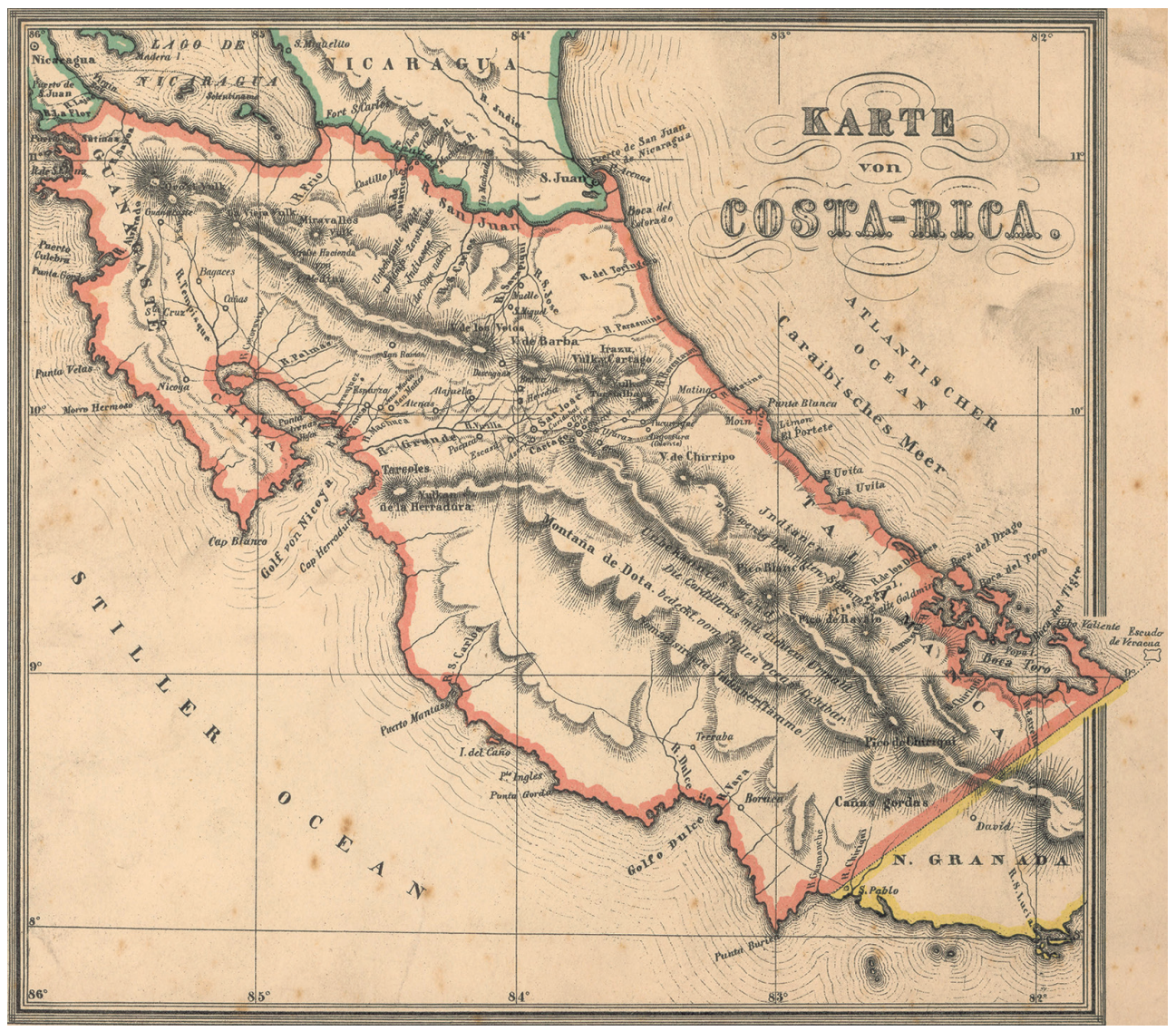

Figura 1. Mapa denominado Karte von Costa Rica, de 1854. Reproducción fotolitográfica de la edición de Wagner U. Scherzer, titulada Costa Rica, publicada en Leipzig en 1856. Tomado de Colección mapas y planos, de la Dirección General del Archivo Nacional de Costa Rica (signatura 8850), Costa Rica. Reproducido con permiso. 
público y privado a través del Open Access y el software libre para la toma de decisiones (Vardakosta y Kapidakis, 2013).

Entre los hallazgos que han sido identificados en la colección ya digitalizada, se encuentran mapas de todo el país, también ejemplares regionales y de otras latitudes. Dentro de las temáticas abarcadas están las cartografías de líneas vitales (acueductos, carreteras, líneas de transmisión, líneas ferroviarias), de centros urbanos, mapas político-administrativos, de recursos naturales, de actividades agropecuarias (café, banano y otros), de límites internacionales, cartas topográficas, entre otros.

Uno de los mapas de Costa Rica que más llaman la atención es el realizado por Wagner U. Scherzer en 1856. Este documento se relaciona con el periodo colonial donde indica la división entre el Virreinato de Nueva España y Nueva Granada. También es interesante, entre otros aspectos, el límite recto entre estos dos territorios, la imprecisión de las líneas de costa y la manera de fijar el límite entre Costa Rica y Nicaragua. Se hace énfasis en la localización de la población en el país, donde se destaca el Valle Central y algunos sectores muy específicos (Guanacaste, Puntarenas y Matina, por ejemplo), además de un limitado poblamiento del sur de la nación.

Un ejemplo de la belleza artística de los mapas realizados a inicios del siglo pasado, es el mapa de la Isla del Caño realizado por D. A. Bejarano en 1915. En este documento se busca la precisión de su localización de acuerdo al sistema de

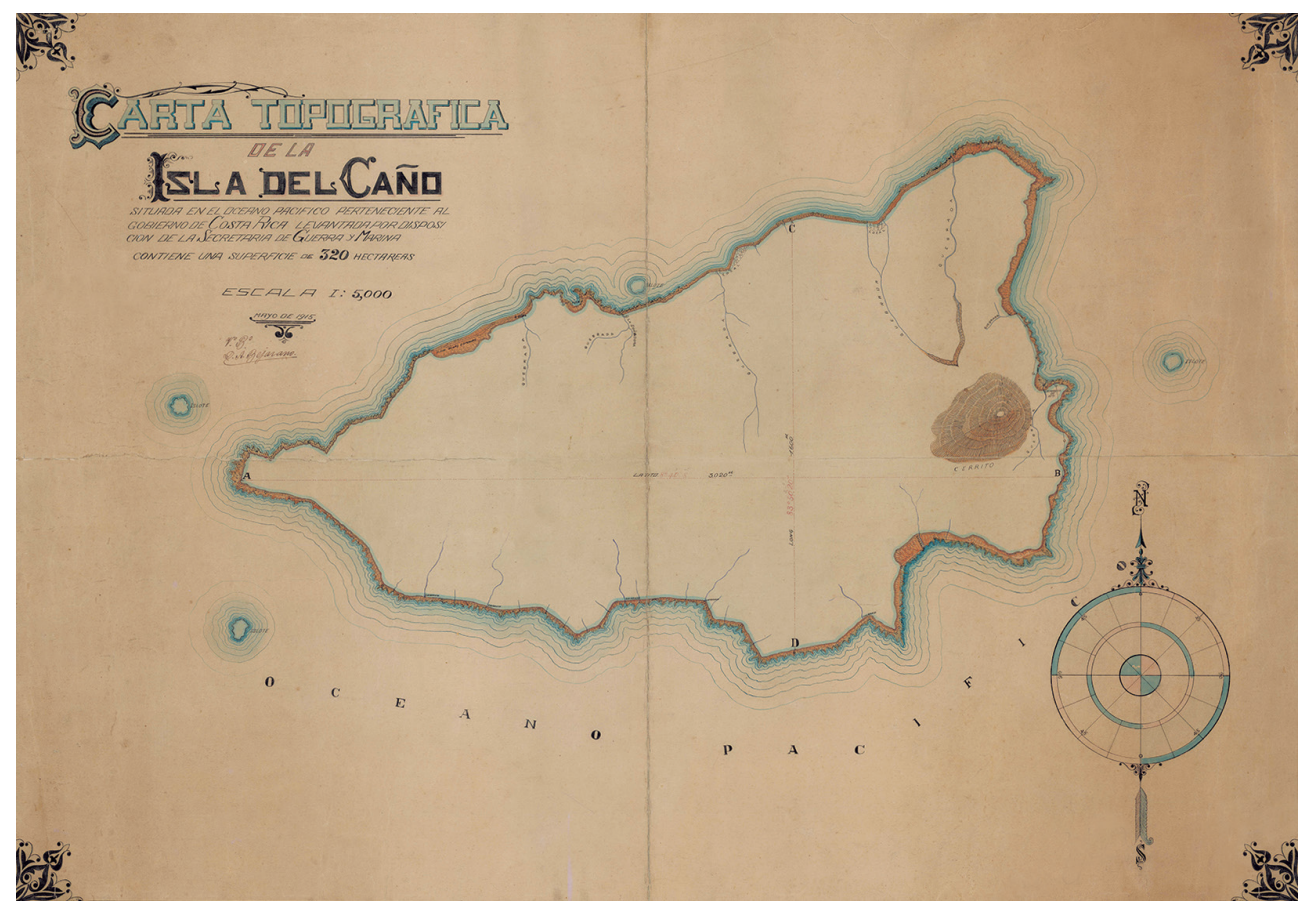

Figura 2. Carta topográfica de la isla del Caño, de 1915. Realizada por D. A. Bejarano para la Secretaría de Guerra y Marina. Escala 1: 5000. Tomado de Colección mapas y planos, de la Dirección General del Archivo Nacional de Costa Rica (signatura 7075), Costa Rica. Reproducido con permiso. 
coordenadas geográficas, la determinación de varios accidentes geográficos como quebradas, cataratas, bahías, un cerro, una playa e islotes. Cabe resaltar la calidad del trazo gracias a la escala utilizada (1:5000) y el detalle del ente público encargado de estos trabajos, la Secretaría de Guerra y Marina.

Entre los resultados publicados a partir del análisis de los mapas escaneados en este convenio, están los atlas histórico-temáticos de diferentes actividades o sucesos del país. Un ejemplo lo constituye el trabajo presentado como resultado de la práctica profesional del Diplomado en Cartografía Digital de la ECG-UNA, titulado Atlas histórico de Costa Rica: Límite fronterizo con Nicaragua (Padilla, Sánchez, Mejía y Castillo, 2013). Este análisis está disponible para su descarga en la página web de la Mapoteca Virtual (www.mapoteca.geo. una.ac.cr/). Este documento fue elaborado a partir de una revisión de los primeros 500 mapas escaneados.

El Atlas histórico de Costa Rica: Límite fronterizo con Nicaragua está dividido en tres secciones acordes con periodos históricos significativos en este proceso de consolidaciones de la frontera norte. El capítulo I corresponde con el periodo 1858-1887, esta sección se introduce con extractos del Tratado de

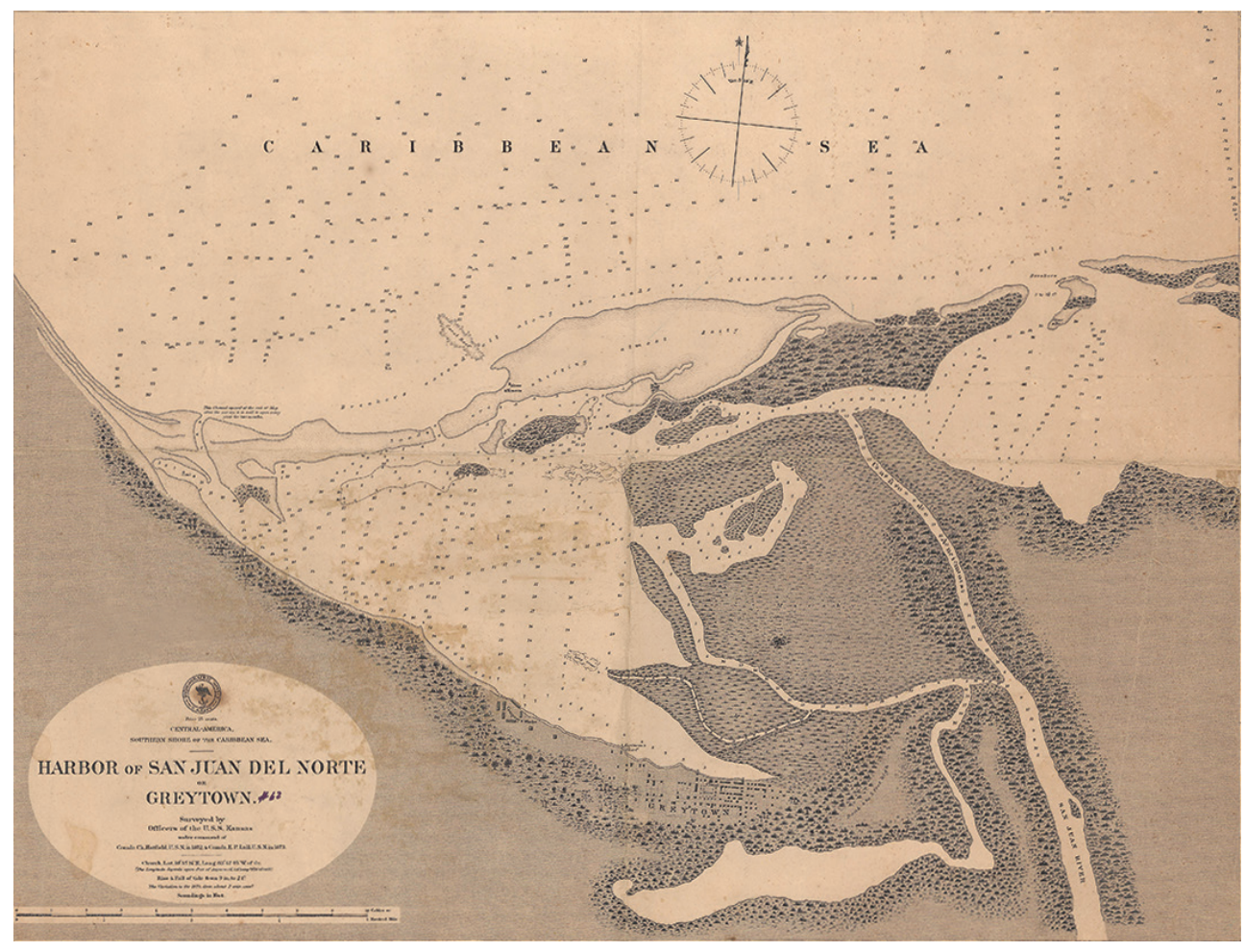

Figura 3. Mapa batimétrico (en pies) del puerto de San Juan del Norte o Greytown, de 1872. Realizado por R. H. Wyman para la Oficina Hidrográfica de la Fuerza Naval de los Estados Unidos y publicado en 1874. Tomado de Colección mapas y planos, de la Dirección General del Archivo Nacional de Costa Rica (signatura 8080), Costa Rica. Reproducido con permiso. 
Límites entre Costa Rica y Nicaragua denominado Cañas-Jerez y se compone por un primer mapa batimétrico (en pies) del puerto de San Juan del Norte o Greytown en 1872, realizado por la Oficina Hidrográfica de la Fuerza Naval de los Estados Unidos. Estos mapas eran comunes a finales del siglo XIX debido a que había intereses geopolíticos y también como medio de conocimiento de los recursos naturales y accidentes geográficos como bahías, golfos y ensenadas, por lo que se llegó a conocer buena parte de las profundidades de las costas a lo largo de América.

El capítulo II corresponde al periodo 1888-1899, el cual es introducido por una explicación de los acontecimientos históricos que dieron paso al desarrollo del Laudo Grover Cleveland; se compone por un mapa que tiene una sección del Laudo a partir de Bahía Salinas (costa pacífica) en 1890; además de otra carta que incluye cómo termina el límite en el sector Caribe, en San Juan del Norte en 1897. Ambos mapas fueron levantados por las comisiones respectivas de cada país para realizar esta cartografía oficial. En este capítulo también se presenta dos mapas con la corrección del límite de acuerdo al Laudo Cleveland donde se le otorgan dos millas a favor de Nicaragua respecto al límite natural del Lago de Nicaragua.

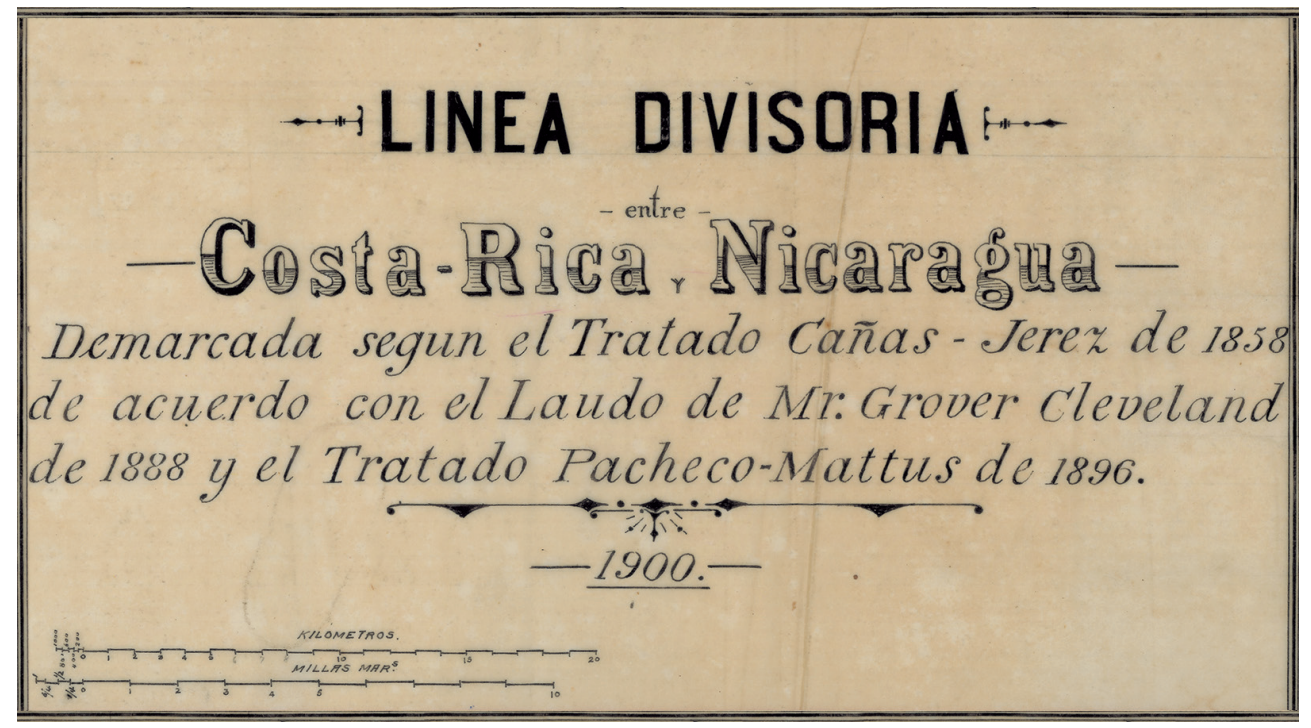

Figura 4. Encabezado del mapa de la línea divisoria entre Costa Rica y Nicaragua en 1900. Rectificación de los límites internacionales, según el Tratado Cañas-Jerez de 1858 de acuerdo con el Laudo Cleveland de 1888 y el Tratado Pacheco-Mattus de 1896. Tomado de Colección mapas y planos, de la Dirección General del Archivo Nacional de Costa Rica (signatura 4175), Costa Rica. Reproducido con permiso.

El capítulo III abarca el periodo de 1900-1970, en él se muestra las Actas XXI, XXII y XX, documentos que aseguraban en 1899 la finalización de la demarcación de mojones para la determinación del límite entre los dos países, en estas actas se indica que el mojón original de punta de Castilla había sido borrado por las fuerzas 


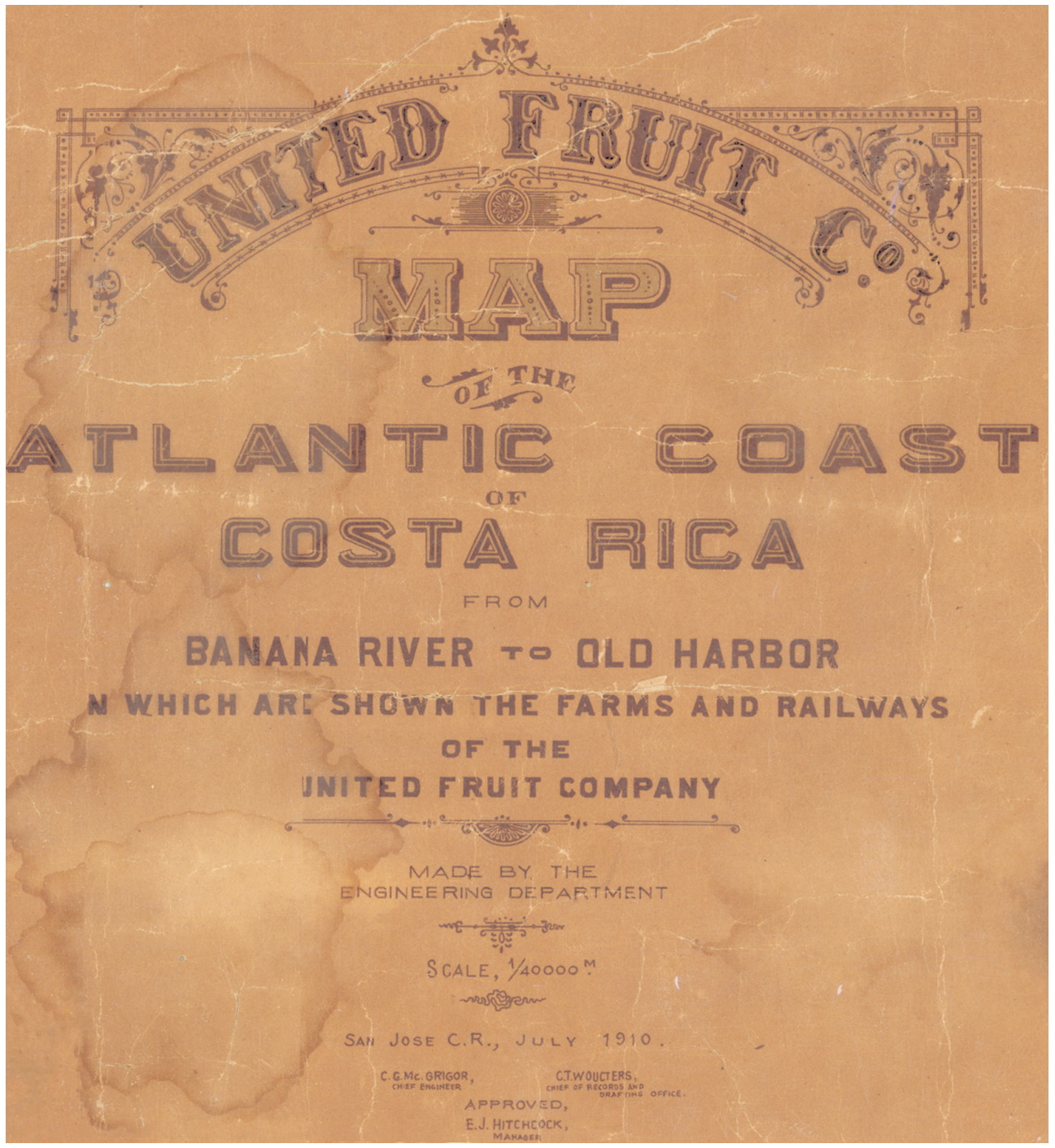

Figura 5. Leyenda de un mapa de las plantaciones bananeras entre el río Banano y el puerto Viejo, propiedad de la UFCo en el Caribe costarricense, 1910. Tomado de Colección mapas y planos, de la Dirección General del Archivo Nacional de Costa Rica (signatura 7168), Costa Rica. Reproducido con permiso.

del mar y que realizarían tres más en mampostería para que resistiera el poder de la erosión costera. Los mapas que componen este capítulo resultan de los mapas finales de los límites internacionales entre Costa Rica y Nicaragua, fijados a partir del Tratado Cañas-Jerez de 1856 y modificados por el Laudo Cleveland de 1888.

El último trabajo que se realizó como práctica profesional supervisada es el Atlas histórico sobre las bananeras en Costa Rica (Peraza-Méndez, 2014), el cual es un acervo cartográfico que incluye un análisis histórico de los dos periodos (explicados en dos capítulos) de la actividad bananera en Costa Rica, tanto en el Caribe (1884-1930) como en el Pacífico Sur (1930-1984). Este representa un valioso aporte 
para el mejor entendimiento espacial del desarrollo de grandes compañías bananeras en Costa Rica como fue la United Fruit Company (UFCo).

En el capítulo I, se explica cuales fueron las razones de la llegada de Henry Meiggs y Minor Keith a Costa Rica para invertir en la actividad bananera, que posteriormente dieron con el establecimiento de la United Fruit Company en la región centroamericana y de la Compañía Bananera de Costa Rica. Además se expone una serie de 19 mapas que muestran las áreas cultivadas con banano en la vertiente

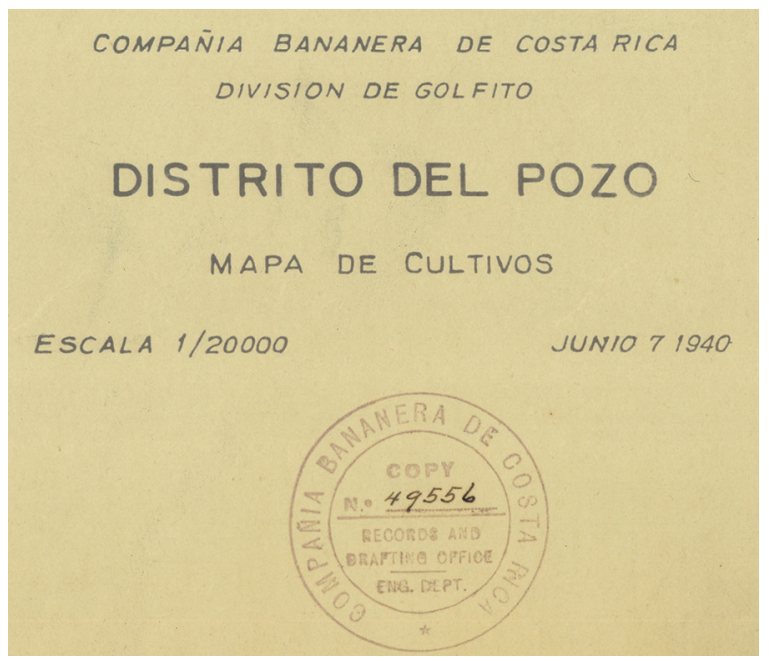

Figura 6. Leyenda de un mapa del distrito del Pozo en la división de Golfito, propiedad de la Compañía Bananera de Costa Rica, 1940. Tomado de Colección mapas y planos, de la Dirección General del Archivo Nacional de Costa Rica (signatura 16488), Costa Rica. Reproducido con permiso.

Caribe; de la misma forma se exhibe como el crecimiento de la producción y la creación del ferrocarril vienen acompañados con el establecimiento de nuevos centros de población como fueron Guácimo, Siquirres, Zent, entre otros.

En el capítulo II, se hace un análisis de la cartografía producto del desarrollo y legado de la actividad bananera en la Zona Sur de Costa Rica. A partir del embate del mal de Panamá en el Caribe, la actividad bananera se traslada al Pacífico Central y Sur, donde se muestran mapas de las divisiones de Quepos (cuencas de los ríos Parrita, Damas, Naranjos y Savegre), división Golfito (delta del río Térraba y de Palmar hasta Golfito) y la división Armuelles (la cual incluía el valle de Coto Colorado en el extremo sureste del país).

\section{CONCLUSIONES}

La importancia de la Mapoteca Virtual como medio para difundir la información cartográfica en el país, radica en su fácil accesibilidad, la originalidad y disposición de los datos para todo tipo de usuarios: desde estudiantes de todos los niveles, académicos, investigadores y la comunidad en general.

El convenio entra la UNA y la DGAN ha permitido el rescate de la colección de mapas y planos que tienen un inestimable valor cultural, no solo para la historia nacional, sino para la geopolítica y la geografía histórica. Los metadatos elaborados y su utilización como índices en los motores de búsqueda a nivel mundial, junto con la posibilidad que brinda el enfoque de Open Access, permitirá que estos documentos 
puedan ser utilizados en una variedad de trabajos que van desde el ámbito escolar hasta la investigación.

El desarrollo de atlas es una muestra del potencial que tiene la información levantada en este proyecto, tales son los casos del Atlas histórico de Costa Rica: Límite fronterizo con Nicaragua y el Atlas histórico sobre las bananeras de Costa Rica, como un par de muestras del uso académico de dichos insumos. Otros tipos de investigación que pueden derivarse de la colección de datos son estudios sobre el desarrollo de centros urbanos, cambios en el uso del suelo, problemas históricos en la disposición de desechos sólidos, la evolución de la red vial de Costa Rica, entre otros.

Es importante resaltar que a pesar del éxito logrado en la digitalización de material cartográfico de incalculable valor histórico, el hecho de que los mapas se encuentren mezclados con planos constructivos de edificios, carreteras y otras obras, hace que el proceso de selección y escaneo sea lento. A esto se suma el mal estado de conservación en que se encuentran algunos mapas que aún no han sido restaurados, la información incompleta en las bases de datos del Archivo Nacional sobre la colección de planos y mapas, la ausencia de algunos materiales de la colección, las omisiones de varios mapas y la falta de presupuesto para el mantenimiento del escáner.

El reto para la sostenibilidad del proyecto está en la adquisición de un nuevo escáner de mayor amplitud y en la inclusión de un presupuesto de apoyo al proyecto por parte del Archivo Nacional para cubrir costos de mantenimiento. A futuro se espera completar la selección y escaneo de la colección completa, así como georreferenciar planos históricos de ciudades y otros mapas, de modo que puedan ser consultados por medio de un geovisualizador.

\section{REFERENCIAS}

Archivo Nacional de Costa Rica. (2014). Reseña histórica. Recuperado de http://www.archivonacional. go.cr/index.php?option=com_content $\&$ view=article $\&$ id=44\&Itemid=63/

Barrantes, G., Tercero, J., Picado, A. y Morera, C. (2010). La Mapoteca Virtual: un ejemplo de colaboración bajo una plataforma de software libre. Revista Geográfica de América Central, (45), 65-76.

Barrantes, G. (2011). Mapoteca Virtual: una herramienta tecnológica en apoyo a la educación. Revista Geográfica de América Central, (47), 1-10.

International Standards Organization. (2014). ISO 19115: Geographic information - Metadata. Recuperado de http://www.iso.org/iso/home/store/catalogue_tc/catalogue_detail.htm?csnumber=53798/

Murillo-Delgado, J. F. (1981). El Archivo Nacional, colección histórica (1881-1981). (Tesis de Licenciatura en Historia). Universidad de Costa Rica, Costa Rica. 
Padilla, K., Sánchez, M., Mejía, R. y Castillo, R. (2013). Atlas Histórico de Costa Rica: Límite Fronterizo con Nicaragua. (Práctica Profesional de Diplomado en Cartografía y Diseño Digital). Universidad Nacional de Costa Rica, Costa Rica.

Peraza-Méndez, D. (2014). Atlas histórico sobre las bananeras de Costa Rica. (Práctica Profesional de Diplomado en Cartografía y Diseño Digital). Universidad Nacional de Costa Rica, Costa Rica.

Quesada-Camacho, J. R. (2001). Historia de la historiografía costarricense, 1821-1940. Costa Rica: Editorial de la Universidad de Costa Rica.

Reinsfelder, T. y Anderson, J. (2013). Observations and Perceptions of Academic Administrator Influence on Open Access Iniciatives. The Journal of Academic Librarianship, (39), 481-487.

Vardakosta, I. y Kapidakis, S. (2013). The new trends for librarians in management of Geographic information. Procedia - Social and Behavioral Sciences, (73), 794-801. 